\title{
Pathogenic and Opportunistic Respiratory Bacteria-Induced Apoptosis
}

\author{
Marcelo Lancellotti ${ }^{1}$, Rafaella Fabiana Carneiro Pereira ${ }^{1}$, Gisele Gentile Cury ${ }^{1}$ and Luciana Maria de Hollanda ${ }^{2}$ \\ ${ }^{1}$ Department of Biochemistry, Institute of Biology, State University of Campinas (UNICAMP); ${ }^{2}$ Department of Microbiology and Immunology, \\ Institute of Biology, State University of Campinas (UNICAMP); Campinas, SP, Brazil
}

\begin{abstract}
Several pathogenic or opportunistic bacteria have the ability to either induce or inhibit host cell apoptosis. The capacity to modulate cell pathways that result in the induction or delay of host cell apoptosis is considered to be an important bacterial virulence mechanism. These processes could be mediated by different host cell signaling pathways that are subverted by the bacteria. Pathogens are able to activate apoptotic proteins, such as caspases, or inactivate anti-apoptotic proteins, such as NFkB and the MAPKKs, or even up-regulate the endogenous receptor/ ligand system that induces apoptosis, generally when the bacteria are bound to the host cell surface. The bacteriainduced apoptotic or anti-apoptotic processes are often related with the fact that the bacteria acquire the ability to reach the host tissues. However, apoptosis is also considered to be a host defense mechanism against infectious agents. Thus, the apoptosis phenomenon plays a central role in host-pathogen interactions.
\end{abstract}

Key-Words: Pathogenic bacteria, opportunistic bacteria, apoptosis, bacteria induce apoptosis, respiratory bacteria.

Apoptosis is defined as cell death activated by an internally controlled suicide program. It is a subtly orchestrated disassembly of cellular components designed to eliminate unwanted cells during embryogenesis in various physiological processes. Bacteria are able to trigger apoptosis through a great variety of mechanisms, including the secretion of compounds such as protein synthesis inhibitors, pore forming proteins, molecules responsible for the activation of the endogenous death machinery in the infected cell, and super antigens. Because many enzymes and signal transduction pathways mediating apoptosis have been extensively described in several recent reviews, we will restrict the present review to molecules started by bacteria involved in apoptosis. The first group of enzymes involved in many forms of apoptosis, including bacteria-induced apoptosis, are the caspases [1]. These host cell cytoplasmatic enzymes are proteases that cleave many cellular proteins, which lead to changes in the membrane that include alterations to the membrane symmetry, mitochondrial changes, and DNA fragmentation. Mitochondria are also key players in apoptosis [2], since they depolarize, swell, and release pro-apoptotic factors during this process. The pro-apoptotic factors include cytochrome $\mathrm{C}$ and the apoptosis inducing factor (AIF) [3]. Cytochrome $\mathrm{C}$ associates with a scaffold protein APAF-1, leading to the activation of caspase 3 and the execution of cell death AIF is able to trigger cell death independent of caspases, by direct translocation to the nucleus and induction of DNA fragmentation [4]. Also, the CD95 or TNF-receptor employs the activation of caspase 8 to trigger further downstream events such as mitochondrial changes and/or caspase 3 activation, whereas primary DNA damage seems to mediate apoptosis Received on 21 February 2009; revised 7 May 2009.

Address for correspondence: Dr. Marcelo Lancellotti. Department of Biochemistry, Institute of Biology, State University of Campinas (UNICAMP), Campinas, São Paulo State, Brazil Telephone: +55(19)3521-6130, Fax: +55(19)3521-6129, e-mail: mlancell@unicamp.br.

The Brazilian Journal of Infectious Diseases 2009;13(3):226-231. (C) 2009 by The Brazilian Journal of Infectious Diseases and Contexto Publishing. All rights reserved. primarily via mitochondria, followed by activation of "execution" caspases. Pro-apoptotic molecules are balanced by anti-apoptotic factors, being the "Inhibitors of Apoptosis" (IAPs) and the Bcl-2-like proteins among the most important anti-apoptotic factors. IAPs are able to associate with and directly inhibit caspases [5] but a regulation by bacterial factors is presently unknown. Bcl-2-like proteins constitute a whole family of proteins comprising pro- and anti-apoptotic members. The Bcl-2 factor, Bcl-xL, and other members prevent apoptosis by many stimuli, whereas Bim, Bax, Bad, and other members promote apoptosis [6]. The mode of action of Bcl-2like proteins is still unclear.

Mechanisms of bacteria-induced apoptosis employing the host machinery will be discussed in the case of Pseudomonas aeruginosa, Neisseria meningitidis, Haemophilus influenza, Legionella pneumophila, Mycobacterium tuberculosis, Streptococcus pneumoniae have been shown to trigger apoptosis. However, we would like to focus on a few bacteria to demonstrate the paradigms of the bacteria-induced activation of the host cell machinery toward apoptosis, as shown in Figure 1.

\section{The Respiratory Bacteria-Induced Apoptosis}

Pseudomonas aeruginosa

A distinct mechanism is employed by Pseudomomas aeruginosa to trigger the cell death of infected host epithelial cells. P. aeruginosa is considered to be one of the most important bacteria in clinical practice because it is resistant to many antibiotics and plays a crucial role in life-threatening infections of immunocompromised patients. However, most important are the pulmonary P. aeruginosa infections of patients with cystic fibrosis. Almost all patients with this disease develop chronic $P$. aeruginosa infections, which cause lung destruction and the patient's premature death. Upon infection of epithelial cells in vitro or in vivo, P. aeruginosa induces up-regulation of the CD95/CD95 ligand on the cell surface [8]. The CD95/CD95 ligand system is one of the most important endogenous receptor ligand pairs triggering apoptosis. The up-regulation of CD95 and the CD95 ligand on cells infected with $P$. aeruginosa depends on the function of the type III secretion system. When 
Figure 1. The main mechanisms of bacteria induced cell apoptosis. The traced lines represent the cellular plasmatic membrane. The apoptotic pathways induced by Neisseria meningitidis, Mycobacterium tuberculosis, Streptococcus pneumonia, Haemophilus influenza (activation of TLR2/TLR4 and further NFkB production), Pseudomonas aeruginosa (CD45 and caspase 8 activation) and Legionella pneumophila (intracellular pathogen with direct caspase 3 activation). Based in Gao and AbuKwaik [7].

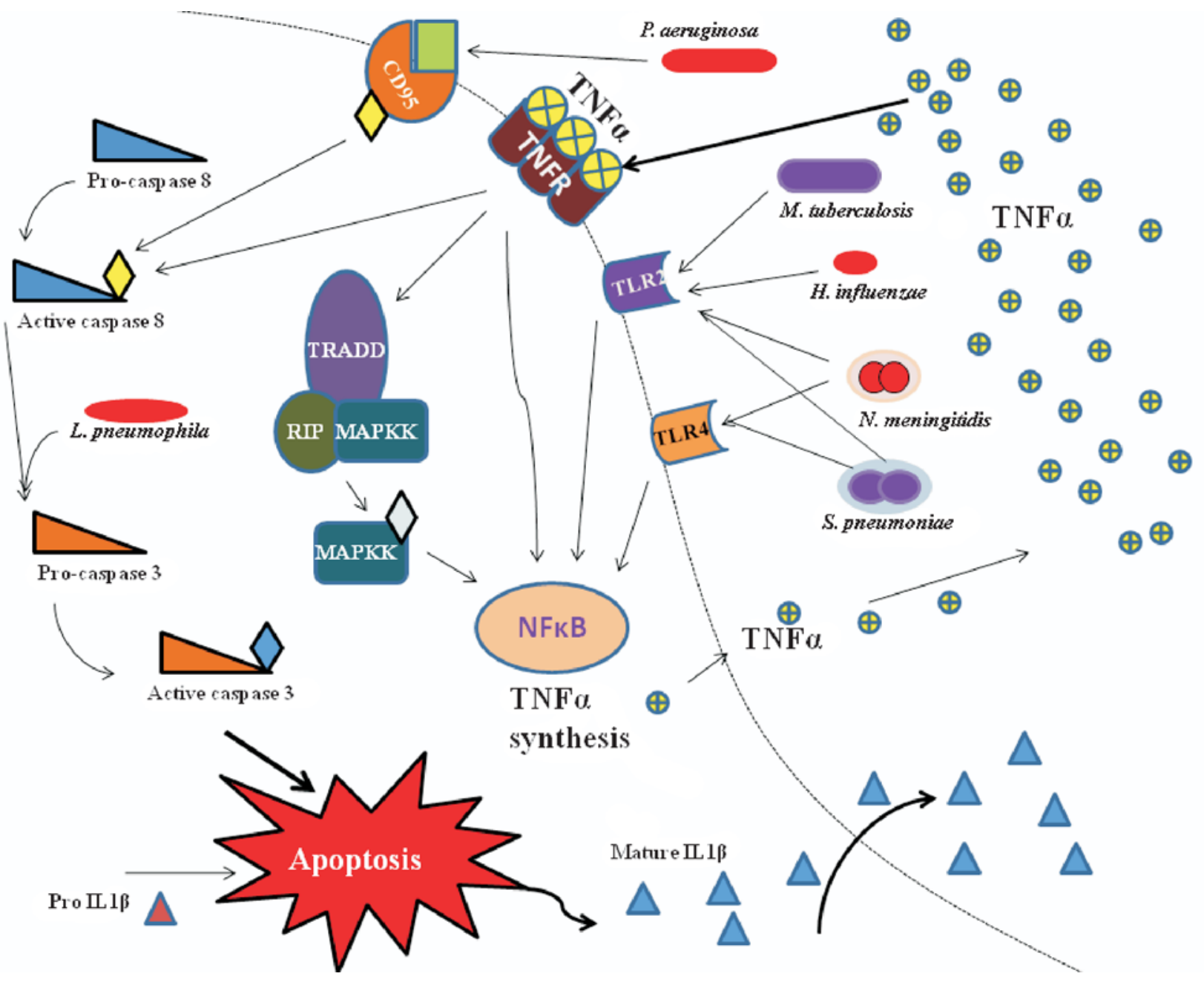

the bacteria lack this secretion system, they almost fail to trigger apoptosis in epithelial cells. Binding of CD95 by the CD95 ligand upon up-regulation induces the activation of caspases 8 and 3 , release of mitochondrial cytochrome C, and JUNK activation. Furthermore, reactive oxygen intermediates seem to be important in the induction of $P$. aeruginosa-triggered death. The significance of the CD95/CD95 ligand system for P. aeruginosatriggered cell death is shown by genetic studies employing cells or mice genetically deficient in functional CD95 or CD95 ligand. In vivo epithelial cells obtained from CD95- or CD95 ligand- deficient mice or ex vivo fibroblasts lacking either CD95 or the CD95 ligand failed to respond to infections by $P$. aeruginosa with an induction of apoptosis [9].
Neisseria meningitidis

The pathogenesis of Neisseria meningitidis and Neisseria gonorrhoeae requires their interaction with human cells and cellular barriers. These pathogens interact with blood, plasma, and exudate fluids, and they also adhere to and invade epithelial and endothelial cells [10-13]. Several Neisseria components are involved in the modulation of pathogenhost cell interactions, including type IV pili, Opa proteins, and porins. There is contrasting information regarding the effects of neisserial porins on apoptosis. Muller et al. [14-16] demonstrated that the $N$. gonorrhoeae porin PorB1B interacts with HeLa cell mitochondria and induces calcium efflux and apoptosis. However, it has been shown that meningococcal 
PorB can interact with mitochondria, thus having a protective effect against the induction of apoptosis by treatment with staurosporine (STS) [17]. There are several explanations for the contrasting effects of $N$. gonorrhoeae PorB1B and $N$. meningitidis PorB on the susceptibility to apoptosis. These include actual differences in the two porins, differences in the purification of the porins, differences in the cell culture conditions (especially the absence or presence of fetal bovine serum in the culture medium) [14-17], and intrinsic differences between the cell lines used in the experiments. The mechanism by which neisserial porins activate lymphocytes B is probably related to their adjuvant activity, which would be attenuated if apoptosis was also induced. Therefore, inhibition of immune cell apoptosis by neisserial porins could be inherent in their immunopotentiating ability. Moreover, because pathogenic Neisseria has been shown to invade host cells, blocking host cell apoptosis might give enough time for the pathogen to adapt to the new environment and multiply to sufficient levels, which will promote further infection. Additionally, the loss of intracellular ions, particularly potassium, plays a primary role in apoptosis, and a deeper understanding of the role of ion channels and plasma membrane transporters in cellular signaling during apoptosis could have important physiological implications for lymphocyte function. This information could be important for the design of therapeutic strategies for several diseases of the immune system, in which apoptosis is involved. Another pathogenic mechanism also involved in Neisseria apoptosis is the meningococcal LOS integrated in the outer membrane, which is released as vesicles or "blebs" from surplus outer membrane material [18,19]. Native outer membrane vesicles (OMVs) contain 24\%-50\% LPS relative to the protein, whereas purified OMVs from $N$. meningitidis, which are partly detoxified so that they can be used for vaccination purposes, contain 5\%9\% LOS relative to their protein content. Recently, two strong candidates for the LOS signaling protein have been identified in myeloid cells and have been termed Toll-like receptor- 2 and 4 (TLR2 and TLR4) [20]. TLR2 is involved in the [21], and bacterial products such as lipopeptides [22]. As for TLR4, it has been shown to mediate the effect of Gram-negative bacteria by LPS [23] together with CD14 [24].

\section{Mycobacterium tuberculosis}

Mycobacterium tuberculosis induces apoptosis in macrophages both in vitro and in vivo via a $\mathrm{TNF} \alpha$ and caspase-1-dependent pathway [25,26]. Both TNF $\alpha$ production by macrophages and induction of apoptosis in macrophages are mediated by binding of the mycobacterial cell wall components and/or lipoproteins to the Toll-like receptor-2 (TLR-2) [27]. However, it is not clear whether these interactions also result in apoptosis, particularly because most pathogenic bacteria have lipoproteins. Nevertheless, some components of the mycobacterial cell wall also influence apoptosis caused by this pathogen. This influence can be exemplified by the lipoarabinomannan - LAM [28,29]. This molecule is able to activate the apoptotic cycle throughout phosphorylation of the Bad protein, preventing binding to the anti-apoptotic proteins $\mathrm{Bcl} 2$ and $\mathrm{BclX}$ [30].

M. tuberculosis also protects cells against apoptosis via two key pathways: induction of the TLR-2-dependent activation of the NFkB cell survival pathway and enhancement of the production of the soluble TNF receptor 2 (sTNFR2), which neutralizes the pro-apoptotic activity of TNF $\alpha$ [31,32]. Therefore, modulation of the apoptotic pathways by $M$. tuberculosis is complex and includes both the induction of cell-death and cell-survival signals. Whether the pro- and antiapoptotic activities are manifested during different stages of the infection is unknown. The modulation of apoptosis by $M$. tuberculosis and its direct and overlapping effects on the immune system are likely to play key roles in pathogenesis.

Macrophage apoptosis occurs within the granuloma. This histological process seems to favor host immunity, but $M$. tuberculosis is capable of partially suppressing it. The proand anti-apoptotic activities of $M$. tuberculosis, which are possibly manifested during different stages of the infection, could be necessary for the establishment of a persistent infection. Vaccines and immunotherapies that result in increased levels of macrophage apoptosis within the granuloma may be able to tip the balance away from the $M$. tuberculosis virulence toward a successful host immune response. Alternatively, increased knowledge of the signaling pathways and effector mechanism(s) triggered during apoptosis should lead to new immunotherapies that could stimulate macrophages to kill M. tuberculosis [30].

In conclusion, apoptosis induction by $M$. tuberculosis is divided into three main events, the arrest under phagosomal maturation (performed by the calcium ions and the cytoplasmatic protein- calmodulin), the anti-apoptotic response (where the Bcl proteins are involved), and the suppression of host cell antibacterial response (role played by MAPKK) [33]. Thus, Mycobacterium species are well adapted to the hostile environment of phagocytic cells, and they use several survival strategies within host cells not seen in the case of other bacteria. A clear understanding of the interaction mechanisms between mycobacteria and host cells still has to be gained.

\section{Legionella pneumophila}

The agent of the Legionnaire's disease, Legionella pneumophila, invades and replicates within the alveolar macrophages, monocytes and, possibly, alveolar epithelial cells. In vitro studies have shown that $L$. pneumophila, as well as Legionella micdadei, induces apoptosis in macrophages and alveolar epithelial cells. The expression of apoptosis-inducing factor(s) by L. pneumophila seems to be regulated by the Dot/ Icm type IV-like secretion system, because several dot/icm mutants fail to induce apoptosis. Thus, L. pneumophila-induced apoptosis via cell contact might be mediated by binding of the pathogen to the common receptor on macrophages and epithelial cells, or via translocation of an effector protein through the Dot/ Icm secretion system [7,34-37]. Although the induction of macrophage apoptosis by L. pneumophila is a constitutive event, 
necrosis mediated by the Legionella pore-forming toxin is temporarily triggered upon termination of bacterial proliferation [7,34-37]. This could represent a coordinated strategy employed by this intracellular pathogen to invade, proliferate within, and eventually exit from the spent host cell. It is possible that $L$. pneumophila-induced apoptosis plays a role in intracellular trafficking and evasion of endocytic fusion, at least during the early stages of the infection. L. pneumophila completely blocks maturation of its phagosome along the endosomal-lysosomal degradation pathway [38]. Apoptosis induced by some facultative intracellular bacteria that block endocytic fusion (such as Legionella) could play a role in modulating the biogenesis of their vacuoles into idiosyncratic niches permissive for intracellular proliferation. It is noteworthy that the ability of $L$. pneumophila to proliferate intracellularly and kill the host cell by apoptosis and necrosis in in vitro tissue culture does not go checked in vivo. A large proportion of Legionnaire's disease patients are immunocompromised, and several host immune responses are activated and are effective against infection by L. pneumophila. Therefore, although L. pneumophila seems to be an uncontrolled pathogen under in vitro experimental conditions, its fate in vivo is controlled at complex levels, which presumably involves both bacterial and host effectors mechanisms, such as INF $\gamma$. During the early stages of infection and the exponential replication of $L$. pneumophila, bacteria induce the activation of caspase-3 by a Dot/Icm-dependent process without driving the infected cell into apoptotic death. At any infection stage, intracellular replication is ceased when apoptosis is triggered in the host cell either by $L$. pneumophila or via caspase-3 activation by pharmacological agents. Also, high caspase-3 activation is observed throughout the pathogen exponential intracellular replication. Caspase-3 binds to the apoptotic cell in the late stages of infection, concomitant with the termination of intracellular replication [39].

Nevertheless, it is known that amoebae cells are the natural hosts of L. pneumophila in the environment [38]. L. pneumophila does not induce apoptosis in amoebae, although amoebae are capable of undergoing apoptosis upon proper stimulation. In contrast with the biphasic killing of mammalian cells, L. pneumophila kills amoebae during the late stages of the infection exclusively via necrosis mediated by poreforming toxins. This mode of killing is essential for the bacteria to egress the protozoan host upon termination of the replication [35]. L. pneumophila disrupts the phagosomal membrane and becomes cytoplasmic at the last stages of infection in both macrophages and Acanthamoeba polyphaga. Lysosomal elements, mitochondria, cytoplasmatic vesicles, and amorphous material are all dispersed among the bacteria after phagosomal disruption, within both human macrophages and A. polyphaga [40].

\section{Haemophilus influenzae}

In non-typeable Haemophilus influenzae (NTHi) is described as involved in inducing-apoptosis in host by induction of epidermal growth factor receptor (EGFR). The EGFR has an important role in regulating human Toll-like receptors
(TLRs), however, it remains unknown. The TLR2 plays a crucial role in host defense against both Gram-positive and -negative bacteria. Although under physiological conditions TLR2 is expressed at a low level in epithelial cells, its expression is greatly up-regulated during bacterial infections via an NF-אB-dependent manner [41-43]. The finding that TLR2 is greatly induced from low to high levels during bacterial infection may have several important implications in host defense and immune response against bacteria. First, the very low expression of TLR2 we observed in unstimulated epithelial cells is likely to be an important aspect of TLR2 function because under limiting conditions the cellular responses to pathogen-associated molecular patterns could be more stringently regulated by controlling the amounts of TLR protein produced [44]. Second, the increased TLR2 expression will probably contribute to the accelerated immune response by epithelial cells as well as the resensitization of epithelial cells to invading pathogens. If so, tight regulation of TLR2 expression should be one of the important immune-regulatory mechanisms commonly involved in host defense against many bacterial strains [45]. Despite the extensive studies on the roles of TLR in host defense and immune response, how the expression of TLR during bacterial infections is tightly regulated still remains largely unknown. Given the important role that EGFR plays in mediating physiological cellular responses such as proliferation and differentiation, its role in regulating pathological cellular response such as host defense and immune response also remains poorly understood [46-49]. The role of EGFR signaling in regulating TLR, the host defense receptor, is totally unclear. Mikami et al. [50] showed for the first time that EGFR acts as a negative regulator for TLR2 induction by the bacterium nontypeable Haemophilus influenzae (NTHi) in vitro and in vivo. The negative regulation of TLR2 induction by EGFR is mediated via an Src-MKK3/6p38 $\alpha / \beta$ MAP kinase-dependent mechanism. Moreover, direct activation of EGFR signaling by the bacterium NTHi-derived EGF-like factor appears to be responsible for triggering the downstream Src-MKK3/6-p38 MAPK signaling, which in turn leads to the negative regulation of TLR2 induction. Finally, exogenous EGF increases NTHi invasion of host epithelial cells, thereby demonstrating the biological significance of TLR2 regulation by EGFR signaling [50].

\section{Streptococcus pneumoniae}

Streptococcus pneumoniae is one of the most common and most aggressive causes of pneumonia and sepsis, and it represents one of the most frequent and most disastrous pathogens in bacterial meningitis. A major exotoxin of Streptococcus pneumoniae is pneumolysin [51]. This toxin colocalized with mitochondrial membranes, altered the mitochondrial membrane potential, and caused the release of apoptosis-inducing factor and cell death. Also, proinflammatory effects of pneumolysin include complement activation, enhanced activation of neutrophils, and increased production of proinflammatory mediators such as tumor necrosis factor alpha, interleukin-1á, and nitric oxide [52]. Pneumolysin activates

www.bjid.com.br 
the innate immune system through Toll-like receptor 4 (TLR4) [53] and described as induced neuronal apoptosis without activating caspase- $1,-3$, or -8 . However, the pneumolysin functions as a mitochondrial toxin is considered as a determinant of caspase-independent apoptosis [21].

Other pneumococci virulence factors are involved in cell induced-apoptosis as the capsular polysaccharide [polysaccharide capsule decreases phagocytosis dependent macrophage apoptosis, the level of which is directly proportional to the number of intracellular bacteria [54]. For bronchial epithelial cells [55,56] and endothelial cells [55], an unencapsulated mutant induces apoptosis but its capsulated parental strain induces necrosis. Polysaccharide capsule also inhibits dendritic cell apoptosis following exposure to pneumococci [57].

Also the pneumococcal cell wall, may mediate apoptosis in brain microvascular endothelial cells causes a delayed caspase-dependent TLR2-mediated apoptosis [58]. Similar mechanisms are likely to explain the late-onset apoptosis which is a feature of exposure of dendritic cells to extracellular subcapsular pneumococcal components and heat-killed bacteria [57]. In macrophages there exists a low-level of apoptosis following exposure to non-opsonised largely extracellular bacteria [54]. Still, the production hydrogen peroxide by the pneumococcus induces cell death in neurons, microglia and brain microvascular endothelial cells [21,58-60] also studied a variety of other pneumococcal factors in the induction of microglial apoptosis and found no role for choline binding protein $\mathrm{A}$, autolysin, surface IgA protease, zinc metalloprotease, pneumococcal surface antigen $\mathrm{A}$ or the comA gene involved in DNA transformation [60]. In monocyte-derived macrophages autolysin, neuraminidase or hyaluronidase did not influence apoptosis induction [54].

\section{Concluding Remarks}

In this review we have described the main differences and similarities encountered in the mechanisms used by bacteria to induce apoptotic or anti-apoptotic effects in host cells. These mechanisms were explored by comparing the apoptosis pathway in host cells by facultative intracellular (Legionella and Mycobacterium), and extra cellular bacteria (Pseudomonas, Neisseria meningitidis, Haemophilus influenzae and Streptococcus pneumoniae). The main events triggered by the microorganisms discussed here are specific for each pathogen and could be summarized in the activation of caspases, mithocondrial alterations, and the action of the MAPK kinases.

Knowledge of these processes could give rise to ways of inhibiting the apoptotic process and provide us with new tools for treating several diseases caused by bacterial pathogens. This could decrease or eliminate the damages caused by these bacteria in the infected host. Thus, elucidation of the major mechanisms involved in the induction of apoptosis; i.e., the signaling pathways, the cellular receptors and/or the bacterial factors involved in this process could reveal new targets for therapy with the purpose of blocking the apoptotic event. For this reason, apoptosis should be considered a major theme in cellular microbiology and in infectious diseases, thus deserving further studies.

\section{Acknowledgements}

This work was supported by CNPq - Post-Doctoral Fellowship to Marcelo Lancellotti Process number 150800/ 2005-3 and FAPESP Process number: 2006/06058-7.

\section{References}

1. Schmitz I., Kirchhoff S., Krammer P.H. Regulation of death receptor-mediated apoptosis pathways. Int $\mathrm{J}$ Biochem Cell Biol 2000;32(11-12):1123-36.

2. Kroemer G., Dallaporta B., Resche-Rigon M. The mitochondrial death/life regulator in apoptosis and necrosis. Annu Rev Physiol 1998;60:619-42.

3. Liu W.W., Mattingly R.R., Garrison J.C. Transformation of Rat-1 fibroblasts with the $\mathrm{V}$-src oncogene increases the tyrosine phosphorylation state and activity of the alpha subunit of Gq/G11. Proc Natl Acad Sci USA 1996;93(16):825863.

4. Joza N., Susin S.A., Daugas E. et al. Essential role of the mitochondrial apoptosis-inducing factor in programmed cell death. Nature 2001;410(6828):549-54.

5. Yang Y.L., Li X.M. The IAP family: endogenous caspase inhibitors with multiple biological activities. Cell Res 2000;10(3):169-77.

6. O’Connor L., Strasser A. The Bcl-2 protein family. Results Probl Cell Differ 1999;23:173-207.

7. Gao L.Y., Abu Kwaik Y. Activation of caspase 3 during Legionella pneumophila-induced apoptosis. Infect Immun 1999;67(9):4886-94.

8. Grassme H., Kirschnek S., Riethmueller J. et al. CD95/CD95 ligand interactions on epithelial cells in host defense to Pseudomonas aeruginosa. Science 2000;290(5491):527-30.

9. Kirschnek S., Paris F., Weller M. et al. CD95-mediated apoptosis in vivo involves acid sphingomyelinase. J Biol Chem 2000;275(35):27316-23.

10. McGee Z.A., Stephens D.S., Hoffman L.H. et al. Mechanisms of mucosal invasion by pathogenic Neisseria. Rev Infect Dis 1983;5(Suppl 4):S708-14.

11. van Putten J.P., Duensing T.D., Carlson J. Gonococcal invasion of epithelial cells driven by P.IA, a bacterial ion channel with GTP binding properties. J Exp Med 1998a;188(5):941-52.

12. van Putten J.P., Duensing T.D., Cole R.L. Entry of OpaA+ gonococci into HEp-2 cells requires concerted action of glycosaminoglycans, fibronectin and integrin receptors. Mol Microbiol 1998b;29(1):369-79.

13. van Putten J.P., Hayes S.F., Duensing T.D. Natural proteoglycan receptor analogs determine the dynamics of Opa adhesinmediated gonococcal infection of Chang epithelial cells. Infect Immun 1997;65(12):5028-34.

14. Muller A., Gunther D., Brinkmann V. et al. Targeting of the proapoptotic VDAC-like porin (PorB) of Neisseria gonorrhoeae to mitochondria of infected cells. Embo J 2000;19(20):5332-43.

15. Muller A., Gunther D., Dux F. et al. Neisserial porin (PorB) causes rapid calcium influx in target cells and induces apoptosis by the activation of cysteine proteases. Embo J 1999;18(2):339-52.

16. Muller A., Rassow J., Grimm J. et al. VDAC and the bacterial porin PorB of Neisseria gonorrhoeae share mitochondrial import pathways. Embo J 2002;21(8):1916-29.

17. Massari P., Ho Y., Wetzler L.M. Neisseria meningitidis porin PorB interacts with mitochondria and protects cells from apoptosis. Proc Natl Acad Sci USA 2000;97(16):9070-5.

18. Devoe I.W., Gilchrist J.E. Release of endotoxin in the form of cell wall blebs during in vitro growth of Neisseria meningitidis. J Exp Med 1973;138(5):1156-67.

19. Melancon J., Murgita R.A., Devoe I.W. Activation of murine B lymphocytes by Neisseria meningitidis and isolated meningococcal surface antigens. Infect Immun 1983;42(2):471-9. 
20. Yoshimura A., Lien E., Ingalls R.R. et al. Cutting edge: recognition of Gram-positive bacterial cell wall components by the innate immune system occurs via Toll-like receptor 2. J Immunol 1999;163(1):1-5.

21. Braun J.S., Hoffmann O., Schickhaus M. et al. Pneumolysin causes neuronal cell death through mitochondrial damage. Infect Immun 2007;75(9):4245-54.

22. Brightbill H.D., Libraty D.H., Krutzik S.R. et al. Host defense mechanisms triggered by microbial lipoproteins through tolllike receptors. Science 1999;285(5428):732-6.

23. Chow J.C., Young D.W., Golenbock D.T. et al. Toll-like receptor4 mediates lipopolysaccharide-induced signal transduction. J Biol Chem 1999;274(16):10689-92.

24. Wright E.K., Goodart S.A., Growney J.D. et al. Naip5 affects host susceptibility to the intracellular pathogen Legionella pneumophila. Curr Biol 2003;13(1):27-36.

25. Keane J., Balcewicz-Sablinska M.K., Remold HG et al. Infection by Mycobacterium tuberculosis promotes human alveolar macrophage apoptosis. Infect Immun 1997;65(1):298-304.

26. Rojas M., Olivier M., Gros P. et al. TNF-alpha and IL-10 modulate the induction of apoptosis by virulent Mycobacterium tuberculosis in murine macrophages. J Immunol 1999;162(10):6122-31.

27. Aderem A., Underhill D.M. Mechanisms of phagocytosis in macrophages. Annu Rev Immunol 1999;17:593-623.

28. Nigou J., Gilleron M., Rojas M. et al. Mycobacterial lipoarabinomannans: modulators of dendritic cell function and the apoptotic response. Microbes Infect 2002;4(9):945-53.

29. Rojas M., Garcia L.F., Nigou J. et al. Mannosylated lipoarabinomannan antagonizes Mycobacterium tuberculosis-induced macrophage apoptosis by altering $\mathrm{Ca}+2$-dependent cell signaling. J Infect Dis 2000;182(1):240-51.

30. Briken V., Porcelli S.A., Besra G.S. et al. Mycobacterial lipoarabinomannan and related lipoglycans: from biogenesis to modulation of the immune response. Mol Microbiol 2004;53(2):391-403.

31. Balcewicz-Sablinska M.K., Keane J., Kornfeld H. et al. Pathogenic Mycobacterium tuberculosis evades apoptosis of host macrophages by release of TNF-R2, resulting in inactivation of TNF-alpha. J Immunol 1998;161(5):2636-41.

32. Kornfeld H., Mancino G., Colizzi V. The role of macrophage cell death in tuberculosis. Cell Death Differ 1999;6(1):71-8.

33. Koul A., Herget T., Klebl B. et al. Interplay between mycobacteria and host signalling pathways. Nat Rev Microbiol 2004;2(3):189-202.

34. Gao L.Y., Kwaik Y.A. The mechanism of killing and exiting the protozoan host Acanthamoeba polyphaga by Legionella pneumophila. Environ Microbiol 2000;2(1):79-90.

35. Gao L.Y., Susa M., Ticac B. et al. Heterogeneity in intracellular replication and cytopathogenicity of Legionella pneumophila and Legionella micdadei in mammalian and protozoan cells. Microb Pathog 1999;27(5):273-87.

36. Gao L.Y., Abu Kwaik Y. Apoptosis in macrophages and alveolar epithelial cells during early stages of infection by Legionella pneumophila and its role in cytopathogenicity. Infect Immun 1999;67(2):862-70.

37. Muller A., Hacker J., Brand B.C. Evidence for apoptosis of human macrophage-like HL-60 cells by Legionella pneumophila infection. Infect Immun 1996;64(12):4900-6.

38. Kwaik Y.A. Fatal attraction of mammalian cells to Legionella pneumophila. Mol Microbiol 1998;30(4):689-95.

39. Abu-Zant A., Santic M., Molmeret M. et al. Incomplete activation of macrophage apoptosis during intracellular replication of Legionella pneumophila. Infect Immun 2005;73(9):5339-49.

40. Molmeret M., Bitar D.M., Han L. et al. Cell biology of the intracellular infection by Legionella pneumophila. Microbes Infect 2004;6(1):129-39.

41. Imasato A., Desbois-Mouthon C., Han J. et al. Inhibition of p38 MAPK by glucocorticoids via induction of MAPK phosphatase- 1 enhances nontypeable Haemophilus influenzae-induced expression of toll-like receptor 2. J Biol Chem 2002;277(49):47444-50.
42. Jono H., Lim J.H., Chen L.F. et al. NF-kappaB is essential for induction of CYLD, the negative regulator of NF-kappaB: evidence for a novel inducible autoregulatory feedback pathway. J Biol Chem 2004;279(35):36171-4.

43. Shuto T., Imasato A., Jono H. et al. Glucocorticoids synergistically enhance nontypeable Haemophilus influenzae-induced Tolllike receptor 2 expression via a negative cross-talk with p38 MAP kinase. J Biol Chem 2002;277(19):17263-70.

44. Jain V., Halle A., Halmen K.A. et al. Phagocytosis and intracellular killing of MD-2 opsonized Gram-negative bacteria depend on TLR4 signaling. Blood 2008;111(9):4637-45.

45. Davey M., Liu X., Ukai T. et al. Bacterial fimbriae stimulate proinflammatory activation in the endothelium through distinct TLRs. J Immunol 2008;180(4):2187-95.

46. Jorissen R.N., Walker F., Pouliot N. et al. Epidermal growth factor receptor: mechanisms of activation and signalling. Exp Cell Res 2003;284(1):31-53.

47. Koziczak-Holbro M., Joyce C., Gluck A. et al. IRAK-4 kinase activity is required for interleukin-1 (IL-1) receptor- and tolllike receptor 7-mediated signaling and gene expression. J Biol Chem 2007;282(18):13552-60.

48. Olayioye M.A., Neve R.M., Lane H.A. et al. The ErbB signaling network: receptor heterodimerization in development and cancer. Embo J 2000;19(13):3159-67.

49. Yarden Y., Sliwkowski M.X. Untangling the ErbB signalling network. Nat Rev Mol Cell Biol 2001;2(2):127-37.

50. Mikami F., Gu H., Jono H. et al. Epidermal growth factor receptor acts as a negative regulator for bacterium nontypeable Haemophilus influenzae-induced Toll-like receptor 2 expression via an Src-dependent p38 mitogen-activated protein kinase signaling pathway. J Biol Chem 2005;280 (43):3618594.

51. Mitchell J.A., Paul-Clark M.J., Clarke G.W. et al. Critical role of toll-like receptors and nucleotide oligomerisation domain in the regulation of health and disease. $J$ Endocrinol 2007;193(3):323-30

52. Zhang Q., Bagrade L., Bernatoniene J. et al. Low CD4 T cell immunity to pneumolysin is associated with nasopharyngeal carriage of pneumococci in children. J Infect Dis 2007;195(8):1194-202.

53. Srivastava A., Henneke P., Visintin A. et al. The apoptotic response topneumolysin is Toll-like receptor 4 dependent and protects against pneumococcal disease. Infect Immun 2005;73(10):6479-87.

54. Ali F., Lee M.E., Iannelli F. et al. Streptococcus pneumoniaeassociated human macrophage apoptosis after bacterial internalization via complement and Fcgamma receptors correlates with intracellular bacterial load. J Infect Dis 2003;188(8):1119-31.

55. N'Guessan P.D., Schmeck B., Ayim A. et al. Streptococcus pneumoniae R6x induced p38 MAPK and JNK-mediated caspase-dependent apoptosis in human endothelial cells. Thromb Haemost 2005;94(2):295-303.

56. Schmeck B., Gross R., N'Guessan P.D. et al. Streptococcus pneumoniae-induced caspase 6-dependent apoptosis in lung epithelium. Infect Immun 2004;72(9):4940-7.

57. Colino J., Snapper C.M. Two distinct mechanisms for induction of dendritic cell apoptosis in response to intact Streptococcus pneumoniae. J Immunol 2003;171(5):2354-65.

58. Bermpohl D., Halle A., Freyer D. et al. Bacterial programmed cell death of cerebral endothelial cells involves dual death pathways. J Clin Invest 2005;115(6):1607-15.

59. Braun J.S., Novak R., Murray P.J. et al. Apoptosis-inducing factor mediates microglial and neuronal apoptosis caused by pneumococcus. J Infect Dis 2001;184(10):1300-9.

60. Braun J.S., Sublett J.E., Freyer D. et al. Pneumococcal pneumolysin and $\mathrm{H}(2) \mathrm{O}(2)$ mediate brain cell apoptosis during meningitis. J Clin Invest 2002;109(1):19-27. 